\title{
Microemulsion Efficiency Boosting and the Complementary Effect. 1. Structural Properties
}

\author{
D. Byelov, H. Frielinghaus,* O. Holderer, J. Allgaier, and D. Richter \\ Forschungszentrum Jülich GmbH, Institut für Festkörperforschung, D-52425 Jülich, Germany
}

Received March 16, 2004. In Final Form: September 6, 2004

\begin{abstract}
Amphiphilic diblock copolymers added to microemulsions proved to enhance the efficiency of surfactants dramatically. The complementary effect of homopolymers is considered in the current work. A possible application of the homopolymer addition could be the viscosity tuning of the microemulsion without changing the considered bicontinuous phase. Furthermore, (homo)polymers are added for many other reasons in technical applications. A theory by Eisenriegler predicts a decreased efficiency when homopolymers are added. In further experiments, the simultaneous addition of homopolymers and diblock copolymers probes whether the two opposite effects superpose and allow for a compensation. Then, efficiency and viscosity are adjustable independently. Experimentally, phase diagrams are investigated and the microscopic structure is measured by small-angle neutron scattering. Within the presented models, both experimental methods are compared and discussed on the basis of the surfactant membrane bending moduli. The homopolymer effect is about 7 times larger than that theoretically predicted, and the superposition of the two polymer effects allows for a compensation with an optionally tunable viscosity.
\end{abstract}

\section{Introduction}

One important additive to microemulsions has been identified in the recent years. ${ }^{1-3}$ Small amounts of amphiphilic diblock copolymer added to nonionic surfactants significantly enhance the solubilization efficiency of the resulting surfactant-polymer mixture relative to the pure surfactant. Accordingly, the total fraction needed to form thermodynamically stable one-phase microemulsions from equal volumes of water and oil is lower if a polymer-surfactant mixture is used instead of the pure surfactant. This means that the efficiency of the surfactant is dramatically enhanced, which was called the "boosting effect" in the literature. ${ }^{1}$ A total amount of $0.5 \mathrm{vol} \%$ polymer increases the efficiency about 5 -fold. In the present study, we investigate how the phase behavior of microemulsions is influenced by the addition of two homopolymer chains made from monomers forming either one of the copolymer blocks, respectively. In our studies, we found a decrease of the surfactant's solubilization efficiency upon homopolymer additions, which is complementary to the efficiency boosting effect upon the addition of amphiphilic block copolymer.

Microemulsions consist of two immiscible components, oil and water, which are mediated by a third component, the surfactant. ${ }^{4}$ The surfactant forms a film between the domains which consist of quite pure oil or water. The domain sizes cover a range from several nanometers to micrometers. ${ }^{5}$ Microemulsions are formed under equilibrium conditions, which distinguishes them from emulsions. One interesting property of microemulsions is the variety of phases with different microstructures. The domains can form many different structures, reaching

(1) Jakobs, B.; Sottmann, T.; Strey, R.; Allgaier, J.; Willner, L.; Richter, D. Langmuir 1999, 15, 6707.

(2) Endo, H.; Allgaier, J.; Gompper, G.; Jakobs, B.; Monkenbusch, M.; Richter, D.; Sottmann, T.; Strey, R. Phys. Rev. Lett. 2000, 85, 102.

(3) Gompper, G.; Endo, H.; Mihailescu, M.; Allgaier, J.; Monkenbusch, M.; Richter, D.; Jakobs, B.; Sottmann, T.; Strey, R. Europhys. Lett. 2001, 56, 683 .

(4) Safran, S. A. Statistical thermodynamics of surfaces, interfaces, and membranes; Addison-Wesley: Reading, MA, 1994.

(5) Sottmann, T.; Muller, S.; Strey, R.; Richter, D.; Allgaier, J. Abs. Pap. Am. Chem. Soc. 2002, 224, 262.

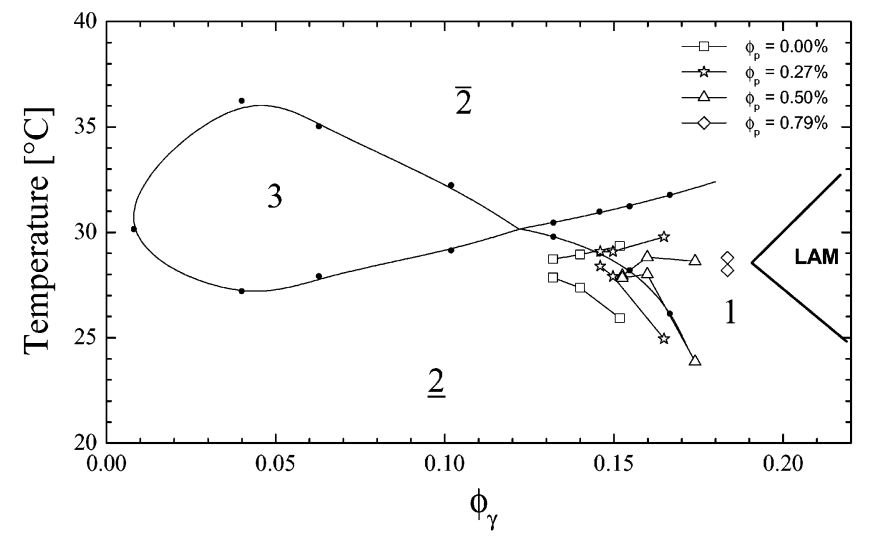

Figure 1. Phase boundaries of the ternary system $\mathrm{H}_{2} \mathrm{O}-$ decane- $\mathrm{C}_{10} \mathrm{E}_{4}$ ("fish diagram": solid line, black filled dots) as a function of temperature and surfactant volume fraction. Volumes of water and decane are equal. For higher homopolymer fractions, $\phi_{\mathrm{p}}$, added to the system $\mathrm{D}_{2} \mathrm{O}$-decane $-\mathrm{C}_{10} \mathrm{E}_{4}$, lines with hollow symbols are used (see legend). The temperature shift of the phase diagram is caused by the use of $\mathrm{D}_{2} \mathrm{O}$ instead of $\mathrm{H}_{2} \mathrm{O}$. The location of the lamellar phase region is estimated by a measurement at $T=28{ }^{\circ} \mathrm{C}$.

from spherical droplets over elongated droplets to bicontinuous structures. Depending on correlations, the microemulsions can be ordered (in a liquid crystalline state) or disordered.

For the discussion of surfactant efficiency, a phase diagram with reduced variables usually is considered. The volumes of oil and water are chosen to be equal. In a three component microemulsion, temperature and surfactant volume fraction remain the two principal variables to be discussed. One obtains a so-called "fish phase diagram" (as obtained in Figure 1). At low (high) temperatures, a microemulsion phase coexists with an oil (water) excess phase. At intermediate temperatures and low surfactant contents, a microemulsion phase coexists with an oil and water excess phase. This region forms the fish body. Upon increasing the surfactant amount, a one-phase microemulsion is achieved. This region forms the fish tail. The crucial point is the fish-tail point where the three-phase 
region touches the one-phase region. The minimum amount of surfactant needed to solubilize equal amounts of oil and water tells about the surfactant efficiency. The ordered phases at even higher surfactant amounts are not considered any further in this study. This phase diagram is also used to discuss the effect of polymer additions under the assumption that the system is quasiternary: ${ }^{1}$ The total amphiphile (surfactant and diblock copolymer) or the aqueous domain (water plus watersoluble polymer) and the oil domain (...) are considered to be one component, respectively.

Besides the diblock copolymers, which are added for efficiency reasons, other polymers are added for various reasons. ${ }^{6,7}$ Homopolymers can inhibit a nondesired highly viscous state of a concentrated surfactant water mixture. ${ }^{6,8}$ The resulting material dissolves much quicker in the final application. Certain polymers are responsible for an antiredeposition effect, ${ }^{7}$ which keeps the contamination away from the cleaned fabric. Enzymes, which are complicated polymers themselves, support either the removal ${ }^{7}$ or the degradation of certain fats and proteins. Certain polyelectrolytes allow one to keep the crystallites of undesired precipitating calcium carbonate as small as possible. ${ }^{7,9}$ The resulting aggregates can be washed out easily.

A recent review ${ }^{10}$ summarizes many polymer aspects in microemulsions. The nonadsorbing polymers mentioned there mainly correspond to the homopolymers considered here. The water-soluble homopolymer causes the coexistence of the lamellar phase with a water (polymer rich) excess phase, which does not appear without polymer. Another study ${ }^{12}$ focuses on the three-phase coexistence and finds an enlarged water phase in which the homopolymer is found. Thus, the homopolymer always seems to drag the water out of the microemulsion phase, which corresponds to a decreased solubilization efficiency. Beyond the polymer as additive, there are interesting results on polymeric microemulsions ${ }^{13-15}$ containing homopolymers and diblock copolymers only.

The polymers of the current study are to be used in small amounts added for tuning the properties of the microemulsion. The concerned additions yield microemulsions with the bicontinuous microstructure of the polymerfree microemulsions being conserved. The boosting effect allows minimization of the required amount of surfactant. Homopolymers can optionally change the overall viscosity. ${ }^{16}$ The effect of homopolymers on the surfactant efficiency is under consideration in the present study.

Previous studies on amphiphilic diblock copolymers were mainly focused on bicontinuous microemulsions. ${ }^{1}$ The increasing efficiency was documented by phase diagram measurements. Using contrast variation mea-

(6) Goddard, E. D. In Interactions of Surfactants with Polymers and Proteins; Goddard, E. D., Ananthapadmanabhan, K. P., Eds.; CRC Press: Boca Raton, FL, 1993

(7) Zini, P. Polymeric additives for high performing detergents; Technomic Publishing Company: Lancaster, PA, 1995.

(8) Brackman, J. C.; Engberts, J. B. F. N. J. Am. Chem. Soc. 1990 112,872 .

(9) Rieger, J.; Thieme, J.; Schmidt, C. Langmuir 2000, 16, 8300.

(10) Sottmann, T. Curr. Opin. Colloid Interface Sci. 2002, 7, 57.

(11) Bagger-Jörgensen, H.; Coppola, L.; Thuresson, K.; Olsson, U.; Mortensen, K. Langmuir 1997, 13, 4204.

(12) Kabalnov, A.; Olsson, U.; Wennerström, H. Langmuir 1994, 10 , 2159.

(13) Washburn, N. R.; Lodge, T. P.; Bates, F. S. J. Phys. Chem. B 2000, 104, 6987 .

(14) Lee, J. H.; Balsara, N. P.; Krishnamoorti, R.; Jeon, H. S.; Hammouda, B. Macromolecules 2001, 34, 6557.

(15) Lee, J. H.; Ruegr, M. L.; Balsara, N. P.; Zhu, Y. Q.; Gido, S. P.; Krishnamoorti, R.; Kim, M. H. Macromolecules 2003, 36, 6537.

(16) Kurata, M.; Tsunashima, Y. In Polymer Handbook; Brandrup, J., Immergut, E. H., Grulke, E. A., Abe, A., Bloch, D. R.; John Wiley and Sons: New York, 1999 (see pages VII/32 and VII/8). surements in a small-angle neutron scattering (SANS) study, two important results were found:2,3 the polymers are anchored in the surfactant film, and the polymers increase the bending rigidity of the surfactant film, which allows the formation of larger bicontinuous structures with lower amounts of surfactant.

The underlying theoretical concept of microemulsion thermodynamics is based on the formulation of Helfrich. ${ }^{17}$ This description assumes that the surface energy of the surfactant film dominates the free energy of the system. The energy has two important contributions arising from the mean curvature and the Gaussian curvature. Two moduli, $\kappa$ and $\bar{\kappa}$, are connected with these curvatures. The bending rigidity, $\kappa$, favors a minimal mean curvature, while a negative saddle splay modulus, $\bar{\kappa}$, favors a minimal genus of the considered surface. In a simplified manner, $\bar{\kappa}$ basically takes the negative value of the bending rigidity. The diblock copolymer influence on $\kappa$ and $\bar{\kappa}$ is described by Lipowski ${ }^{18}$ and favors lower mean curvature. This prediction nicely agrees with the experimentally found boosting effect. The homopolymer influence was predicted by Eisenriegler ${ }^{19}$ and behaves in the opposite way. A decreased efficiency is expected. The experimental challenge concerns the superposition of the two opposite effects. Then, a certain combination of polymers would allow for a constant efficiency with increased viscosity.

This paper compares the macroscopic phase behavior with the microscopic structure observed by SANS experiments. In theory, the phase behavior is connected with the saddle splay modulus, while the structure is connected with the bending rigidity. First, the influence of homopolymers is described, and then, the possible superposition of effects is investigated by simultaneous additions of homopolymers and diblock copolymers. While this paper focuses on the structural properties of a bicontinuous microemulsion with polymer additions, the second paper ${ }^{20}$ concerns the dynamic properties studied by neutron spinecho spectroscopy.

\section{Theory of Microemulsions}

For microemulsions, the dominating term of the free energy concerns the surfactant film only. The domains of oil and water are separated by the surfactant film which behaves like a two-dimensional fluid. The free energy as formulated by Helfrich ${ }^{17}$ then reads

$$
F=\int \mathrm{d} S\left[2 \kappa\left(H-c_{0}\right)^{2}+\bar{\kappa} K+\ldots\right]
$$

The integral over the film surface (eq 1) sums up the local properties of the membrane at each point. The first term considers the deviation of the mean curvature, $H$, from the spontaneous curvature, $c_{0}$. The mean curvature is the average of the two principal curvatures, $H=(1 / 2)\left(c_{1}+c_{2}\right)$. The two principal curvatures are given by the (locally orthogonal) tangential radii in a given point, that is, $c_{i}=$ $1 / R_{i}$, and describe the minimal and maximal curvature. The corresponding modulus, $\kappa$, is the bending rigidity. The next term considers the Gaussian curvature, $K=$ $c_{1} c_{2}$. According to the Gauss -Bonnet theorem, the integral over $K$ is a topological invariant, $4 \pi(n-g)$, where $n$ is the number of separated bodies and $g$ is the genus, that is, the number of holes or handles. The modulus $\bar{\kappa}$ is the saddle splay modulus. In many cases, $\bar{\kappa} \simeq-\kappa$. Our investigations

(17) Helfrich, W. Z. Naturforsch., A: Phys. Sci. 1978, 33, 305.

(18) Hiergeist, C.; Lipowsky, R. J. Phys. II 1996, 6, 1465.

(19) Hanke, A.; Eisenriegler, E.; Dietrich, S. Phys. Rev. E 1999, 59, 6853.

(20) Holderer, O.; Monkenbusch, M.; Byelov, D.; Frielinghaus, H.; Allgaier, J.; Richter, D. Langmuir, manuscript in preparation. 
are focused on the phase inversion temperature where $c_{0}$ changes sign; no curvature is preferred, and typical morphologies are the bicontinuous structure or lamellae.

For the bending rigidity, the theoretical predictions for the contributions from fluctuations and polymer additions are collected:

$$
\begin{array}{rlrl}
\frac{\kappa_{\mathrm{R}}}{k_{\mathrm{B}} T} & =\frac{\kappa_{0}}{k_{\mathrm{B}} T} & \text { pure membrane } \\
& +\frac{\alpha}{4 \pi} \ln (\psi) & & \text { thermal fluctuations } \\
& -0.0238 \frac{\phi_{\mathrm{p}}\left(R_{\mathrm{hW}}^{3}+R_{\mathrm{hO}}^{3}\right)}{V_{\mathrm{p}}} & & \text { homopolymer } \\
& +\frac{1}{12}\left(1+\frac{\pi}{2}\right) \sigma\left(R_{\mathrm{dW}}^{2}+R_{\mathrm{dO}}^{2}\right) & & \text { diblock copolymer }
\end{array}
$$

The pure membrane term describes a genuine property of the surfactant. The thermal fluctuations let the membrane appear floppier on larger length scales. The renormalization prefactor, $\alpha$, theoretically has the value $3 .^{21,22}$ In this term, the reformulation of the length scale in terms of the membrane volume fraction, $\psi$, has been applied. ${ }^{3}$ The next term considers the homopolymer influence according to the theory of Eisenriegler. ${ }^{19}$ The important parameters are the homopolymer volume fraction, $\phi_{\mathrm{p}}$, the end-to-end distances of the homopolymers in either the water, $R_{\mathrm{hW}}$, or the oil phase, $R_{\mathrm{hO}}$, respectively, and the mean volume of a homopolymer molecule, $V_{\mathrm{p}}$. In a similar manner, the diblock copolymer influence is described by the theory of Lipowski. ${ }^{18}$ The important parameters are the grafting density, $\sigma$, of the diblock copolymer on the membrane and the end-to-end distances of the oil- and water-soluble blocks. It is the issue of this work whether all influences superpose in the manner of eq 2 . The same theories yield a similar expression for the saddle splay modulus:

$$
\begin{array}{r}
\frac{\bar{\kappa}_{\mathrm{R}}}{k_{\mathrm{B}} T}=\frac{\bar{\kappa}_{0}}{k_{\mathrm{B}} T}+\frac{\bar{\alpha}}{4 \pi} \ln (\psi)+0.0211 \frac{\phi_{\mathrm{p}}\left(R_{\mathrm{hW}}^{3}+R_{\mathrm{hO}}^{3}\right)}{V_{\mathrm{p}}}- \\
\frac{1}{6} \sigma\left(R_{\mathrm{dW}}^{2}+R_{\mathrm{dO}}^{2}\right)
\end{array}
$$

The prefactors of the polymer related terms are, compared to eq 2 , very similar in magnitude but differ in sign. This means that observable changes of the moduli can be well compared.

The emulsification failure boundary is identified with the transition between the bicontinuous phase and the three-phase coexistence (fish-tail point). Molecular dynamics (MD) simulations ${ }^{23}$ showed that it occurs when the condition $\bar{\kappa}_{\mathrm{R}}=\bar{\kappa}_{\text {FTP }}$ is reached (at $c_{0}=0$ ). The small constant $\bar{\kappa}_{\mathrm{FTP}}$ is not discussed any further here, especially for the undetermined constant $\bar{\kappa}_{0}$, but the influence of the polymers on the minimum membrane fraction, $\psi$, is of importance. Therefore, the macroscopic phase behavior measures changes of the saddle splay modulus by polymer additions.

The microscopic structure of the microemulsion domains is measured by small-angle neutron scattering. The

(21) Peliti, L; Leibler, S. Phys. Rev. Lett. 1985, 54, 1690

(22) David, F. In Statistical Mechanics of Membranes and Surfaces; Nelson, D., Piran, T., Weinberg, S.; World Scientific: Singapore, 1989; pp 157.

(23) Morse, D. C. Phys. Rev. E 1994, 50, R2423. macroscopic cross section, $\mathrm{d} \Sigma / \mathrm{d} \Omega$, as a function of the scattering vector, $Q$, was successfully described by the theory of Teubner and Strey: ${ }^{24-26}$

$$
\frac{\mathrm{d} \Sigma}{\mathrm{d} \Omega}(Q)=\frac{8 \pi\left\langle v^{2}\right\rangle / \xi}{Q^{4}-2\left(k_{0}^{2}-\xi^{-2}\right) Q^{2}+\left(k_{0}^{2}+\xi^{-2}\right)^{4}}
$$

The prefactor $\left\langle v^{2}\right\rangle$ depends on the contrast of the domains, which is given by the scattering length density difference between water $\left(\mathrm{D}_{2} \mathrm{O}\right)$ and oil. The structural parameters are the wave vector $k_{0}=2 \pi / d$ given by the domain size, $d$, and the correlation length, $\xi$. The Teubner-Strey theory results from a Ginzburg-Landau approach. This approach can be matched with the Gaussian random fields theory ${ }^{27-29}$ that contains $\kappa$ as a parameter. Then, the domain size is basically connected with the average membrane surface per unit volume, $k_{0} \sim S / V$, and the correlation length is basically related to the bending rigidity and the surface-to-volume ratio, $\xi \sim \kappa_{\mathrm{R}}(S / V)^{-1}$. The surface-to-volume ratio cancels out when regarding the product:

$$
k_{0} \xi=\frac{64}{5 \sqrt{3}} \frac{\kappa_{\mathrm{R}}}{k_{\mathrm{B}} T} \Theta \underset{\kappa_{\mathrm{R}} \rightarrow \infty}{=} \frac{64}{5 \sqrt{3}} \frac{\kappa_{\mathrm{R}}}{k_{\mathrm{B}} T}
$$

The function $\Theta$ accounts for remaining dependencies on $\kappa_{\mathrm{R}}$ and the surface-to-volume ratio but approaches the limit unity for large bending rigidities. In experiments, ${ }^{3}$ this limit assumedly was reached, but a precise value for an important cutoff parameter is missing. The structural investigations therefore allow measurement of the bending rigidity; that is, the SANS curves that relate to the microscopic structure allow the determination of $\kappa$ from the fitted values for $d$ and $\xi$. The macroscopic phase behavior on the other hand depends on $\bar{\kappa}^{23}$

\section{Experimental Section}

Materials. As oil, we used $n$-decane purchased from Aldrich without further purification. As water, we used heavy water $\left(\mathrm{D}_{2} \mathrm{O}\right)$ from Aldrich without further purification. The surfactant was $n$-decyltetraoxyethylene $\left(\mathrm{C}_{10} \mathrm{E}_{4}\right)$ purchased from either Bachem or Fluka, again, without further purification. The homopolymer poly(ethylene oxide) $\left(\mathrm{PEO}_{5}\right)$ was purchased from Aldrich as a GPC-column standard. The homopolymer poly(ethylene propylene) $\left(\mathrm{PEP}_{5}\right)$ and the diblock copolymer poly(ethylene propylene)poly (ethylene oxide) $\left(\mathrm{PEP}_{5}-\mathrm{PEO}_{5}\right)$ were synthesized by standard anionic polymerization. ${ }^{30}$ The characteristics of the polymers are given in Table 1 . The index of the polymer names $\left(\mathrm{PEP}_{5}, \ldots\right)$ specifies the approximate molar mass in kilograms per mole.

Some specific volume fractions are defined. First of all, the oil-to-water fraction, $\phi_{\mathrm{O}}=V_{\text {oil }}\left(V_{\text {oil }}+V_{\text {water }}\right)$, is chosen to be 0.50 for the whole study. The next important fraction is the surfactant fraction, $\phi_{\gamma}=V_{\text {surf }} /\left(V_{\text {surf }}+V_{\text {oil }}+V_{\text {water }}+V_{\text {hom }}^{\mathrm{O}}+V_{\text {hom }}^{\mathrm{W}}+\right.$ $\left.V_{\text {dibl }}\right)$. This number will finally tell about the efficiency of the surfactant.

The membrane volume fraction, $\psi$, includes the small correction for the surfactant solubility ( $2 \mathrm{vol} \%)$ in the oil phase. The averaged homopolymer fraction in the oil and water domain reads

(24) Teubner, M.; Strey, R. J. Chem. Phys. 1987, 87, 3195.

(25) Mihailescu, M.; Monkenbusch, M.; Endo, H.; Allgaier, J.; Gompper, G.; Stellbrink, J.; Richter, D.; Jakobs, B.; Sottmann, T.; Farago, B. J. Chem. Phys. 2001, 115, 9563.

(26) Endo, H. Ph.D. Dissertation, Universität Münster, Münster, Germany, 2001.

(27) Pieruschka, P.; Safran, S. A. Europhys. Lett. 1993, 22, 625.

(28) Pieruschka, P.; Safran, S. A. Europhys. Lett. 1995, 31, 207.

(29) Endo, H.; Mihailescu, M.; Monkenbusch, M.; Allgaier, J. Gompper, G.; Richter, D.; Jakobs, B.; Sottmann, T.; Strey, R.; Grillo, I. J. Chem. Phys. 2001, 115, 580.

(30) Allgaier, J.; Poppe, A.; Willner, L.; Richter, D. Macromolecules 1997, 30, 1582. 
Table 1. Characteristic Numbers of the Polymers

\begin{tabular}{lclc}
\hline \multicolumn{1}{c}{ polymer } & $M_{\mathrm{n}}{ }^{a}(\mathrm{~g} / \mathrm{mol})$ & $\mathrm{PDI}^{b}$ & $R_{\mathrm{ee}}{ }^{c}(\AA)$ \\
\hline PEP $_{5}$ & 4700 & $<1.025$ & 66 \\
PEO $_{5}$ & 5850 & $<1.025$ & 78 \\
PEP $_{5}-\mathrm{PEO}_{5}$ & 10400 & $<1.03$ & \\
PEP-block $_{\text {PEO-block }}^{4730}$ & $<1.03$ & 66 \\
PEP $_{10}$ & 5700 & & 77 \\
PEO $_{10}$ & 10300 & $<1.04$ & 99 \\
& 10800 & $<1.04$ & 110
\end{tabular}

${ }^{a} M_{\mathrm{n}}$ : degree of polymerization determined by stoichiometry of polymerization. ${ }^{b}$ PDI: polydispersity index $=M_{\mathrm{w}} / M_{\mathrm{n}}$ determined by GPC. ${ }^{c} R_{\text {ee }}$ : end-to-end distances of the blocks calculated by ref 31.

$\phi_{\mathrm{p}}=(1 / 2) V_{\mathrm{hom}}^{\mathrm{O}} /\left(V_{\mathrm{hom}}^{\mathrm{O}}+V_{\text {oil }}\right)+(1 / 2) V_{\mathrm{hom}}^{\mathrm{W}} /\left(V_{\mathrm{hom}}^{\mathrm{W}}+V_{\text {water }}\right)$. In all reported experiments, $V_{\text {hom }}^{\mathrm{O}} /\left(V_{\text {hom }}^{\mathrm{O}}+V_{\text {oil }}\right) \simeq V_{\text {hom }}^{\mathrm{W}} /\left(V_{\text {hom }}^{\mathrm{W}}+V_{\text {water }}\right)$. The possibility of one homopolymer being a cosolvent for the surfactant has been disregarded, since not much is known. Finally, the diblock copolymer fraction measures the polymer content in the total amphiphile, $\phi_{\delta}=V_{\text {dibl }} /\left(V_{\text {dibl }}+V_{\text {surf }}\right)$. All volumes were calculated by the densities of the pure materials assuming ideal mixing. Throughout the paper, we use units of volume fractions.

Small-Angle Neutron Scattering. SANS experiments were carried out on the instrument KWS2 at the FRJ-2 research reactor at the Forschungszentrum Jülich. The wavelength of neutrons is $\lambda=7.3 \AA(\Delta \lambda / \lambda=10 \%)$, and the measurements were performed at three detector/collimation distances, viz., $20 \mathrm{~m} / 20 \mathrm{~m}, 8 \mathrm{~m} / 8 \mathrm{~m}$, and $2 \mathrm{~m} / 4 \mathrm{~m}$; thus, a scattering vector, $Q$, range of $0.002 \AA^{-1} \leq$ $Q \leq 0.2 \AA^{-1}$ was obtained. The scattering data were corrected for background and detection efficiency of the single detector cells, and they were calibrated in absolute units by a secondary Lupolen standard. The macroscopic cross section, $\mathrm{d} \Sigma / \mathrm{d} \Omega$, is obtained in units of inverted centimeters. The use of heavy water as the only deuterated component mainly creates a contrast between the oil and water domains, which is called bulk contrast.

\section{Results}

In this section, results are presented on the bicontinuous microemulsion with (a) addition of homopolymer only and (b) simultaneous addition of homopolymer and diblock copolymer. First, phase diagrams are discussed in the context with predictions of the saddle splay bending modulus, $\bar{\kappa}_{R}$. Second, small-angle neutron scattering measurements probe the microscopic structure. By fitting the Teubner-Strey model, we get two structural parameters: the domain size, $d$, and the correlation length, $\xi$. The expression for the bending rigidity, $\kappa_{\mathrm{R}} \sim \xi / d$, of the Gaussian random field model is finally discussed in the context of theoretical predictions for $\kappa_{\mathrm{R}}$.

Addition of Homopolymers Only. Small added amounts of the two homopolymers $\mathrm{PEP}_{5}$ and $\mathrm{PEO}_{5}$ are considered in this part. The phase diagram (Figure 1) shows the temperature as a function of surfactant content. At low temperatures, there is a two-phase region (2) where an oil-in-water microemulsion coexists with an oil excess phase, and at high temperatures, there is a two-phase region $(\overline{2})$ where a water-in-oil microemulsion coexists with a water excess phase. At intermediate temperatures, one finds one-phase microemulsions (1) for intermediate surfactant mass fractions, a lamellar phase (LAM) for higher surfactant mass fractions, and a three-phase region (3) for lower surfactant mass fractions, where a microemulsion middle phase coexists with oil and water excess phases. Small added amounts of the homopolymers $\mathrm{PEP}_{5}$ and $\mathrm{PEO}_{5}$ increase the minimum quantity of surfactant needed to solubilize water and oil or, in other words, decrease the efficiency of the surfactant (antiboosting effect).

The presence of homopolymer chains in water and oil in the neighborhood of a membrane change the moduli $\kappa_{\mathrm{R}}$

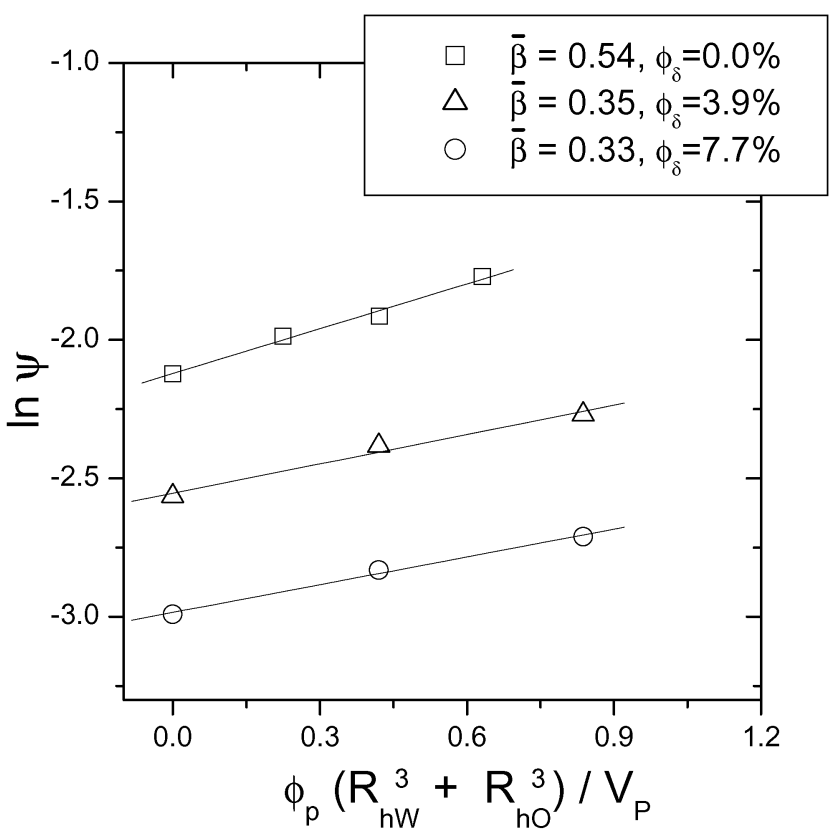

Figure 2. Logarithm of the membrane volume fraction at the fish-tail point as a function of the scaled homopolymer volume fraction. The top data ( $\square$ ) correspond to measurements on homopolymers only and are discussed first (see eq 6). Later discussions involve diblock copolymers simultaneously with the diblock fraction, $\phi_{\delta}$. Slopes of the regression lines are given by $\bar{\beta}$ values.

and $\bar{\kappa}_{R}$ of the membrane. The theory of ideal and selfavoiding polymers near a membran $\mathrm{e}^{19}$ predicts a decrease of the bending rigidity, $\kappa_{\mathrm{R}}$, and an increase of the saddle splay modulus, $\bar{\kappa}_{R}$ (see eqs 2 and 3 ). Such changes of the elastic constants should entail a decrease of efficiency, which will be discussed in the following. At the fish-tail point, the saddle splay modulus, $\bar{\kappa}_{\mathrm{R}}$, is equal to a specific small value, $\bar{\kappa}_{\text {FTP }}$; that is, at the fish-tail point, the net effect of polymer addition, $\Delta \bar{\kappa}$, must be zero. Solving eq 3 for the membrane volume fraction, $\psi$, under this condition yields

$$
\psi=\psi_{0} \exp \left[\bar{\beta} \frac{\phi_{\mathrm{p}}}{V_{\mathrm{p}}}\left(R_{\mathrm{hW}}^{3}+R_{\mathrm{hO}}^{3}\right)\right]
$$

which predicts the effect of homopolymer addition on the membrane volume fraction. $\phi_{\mathrm{p}}$ is the volume fraction of the homopolymers in one domain, $V_{\mathrm{p}}$ is the average molar volume of a homopolymer, and theoretically $\bar{\beta}=0.0795 .{ }^{19}$ $\psi_{0}$ is the membrane volume fraction at the fish-tail point without any polymer.

The phase diagram measurements provide a qualitative proof of this prediction. The fish-tail point moves to higher surfactant contents for every next portion of homopolymer. In Figure 2, the dependence of the logarithm of the membrane volume fraction at the fish-tail point is plotted as a function of the scaled homopolymer number density. This dependence is linear, as follows from eq 6 , but the experimental prefactor $\bar{\beta}=0.54$ describes an approximately 7 times higher sensitivity on the homopolymer addition than that expected from theory. ${ }^{19}$

To extend the study on the bending rigidity, $\kappa_{\mathrm{R}}$, we involved SANS measurements. The samples were measured under bulk contrast. The surfactant volume fraction was in a range between 13 and $20 \mathrm{vol} \%$. The homopolymers $\mathrm{PEP}_{5}$ and $\mathrm{PEO}_{5}$ were added to the oil and water, respectively, in equal amounts with an increment between experiments of $\sim 0.25 \mathrm{vol} \%$. 


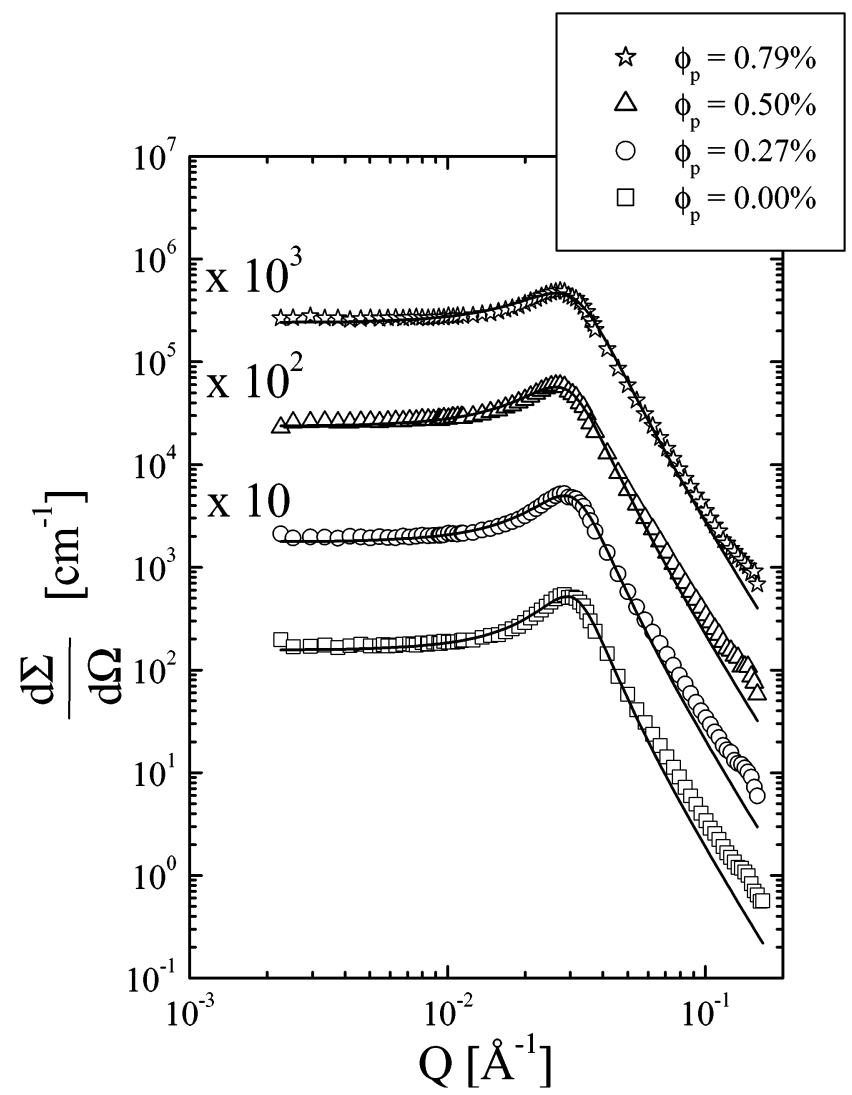

Figure 3. Scattering cross section as a function of the scattering vector for bicontinuous microemulsions with homopolymer volume fractions, $\phi_{\mathrm{p}}$, under bulk contrast. The membrane volume fraction, $\psi$, is fixed at 0.175 . Errors are of the approximate symbol size or below. Solid lines are TeubnerStrey model fittings.

An example of SANS measurements is shown in Figure 3. Here, the macroscopic cross section, $\mathrm{d} \Sigma / \mathrm{d} \Omega$, is depicted as a function of the wave vector, $Q$. The membrane volume fraction $\psi=0.175 \pm 0.003$ for all represented scattering curves was approximately constant. The amount of homopolymers was increased from 0.0 to 0.78 vol \%. For reasons of clarity, the cross sections for every next addition of homopolymer were multiplied by a factor of 10 . The high intensity at low $Q$ indicates large oil/water concentration fluctuations at large scales. In the middle- $Q$ region, a correlation peak exists at $\sim 0.03 \AA^{-1}$. This value is connected with the domain size by the rough estimate $d$ $=2 \pi / Q_{\max }$. The width of this peak is connected with the correlation length. A Porod behavior at high $Q \geq \mathrm{Q}_{\mathrm{P}}=$ $0.05 \AA^{-1}$ is characteristic for the randomly oriented sharp interfaces. Note that the $I \sim Q^{-4}$ asymptote of the Teubner-Strey form is not equal to the Porod $I \sim Q^{-4}$ behavior; $Q_{P}$ separates both regimes. A refined quantitative analysis of the scattering data is based on the Teubner-Strey formula (eq 4), which was used to describe the peak intensity. Fits were limited to $\mathrm{Q}_{\mathrm{P}}$, where the Porod intensity dominates. Examples of fitting curves are shown in Figure 3. From the fitting, the characteristic domain size, $d$, and the correlation length, $\xi$, are obtained. The structure of the microemulsion is characterized.

Now we discuss the domain size, $d$, as a function of the membrane volume fraction, $\psi$, for the samples with different homopolymer contents (see Figure 4). The dependence of $d$ on the homopolymer volume fraction is rather weak compared to the dependence on the membrane volume fraction. The typical structural scale is mainly governed by the amount of surfactant in the system.

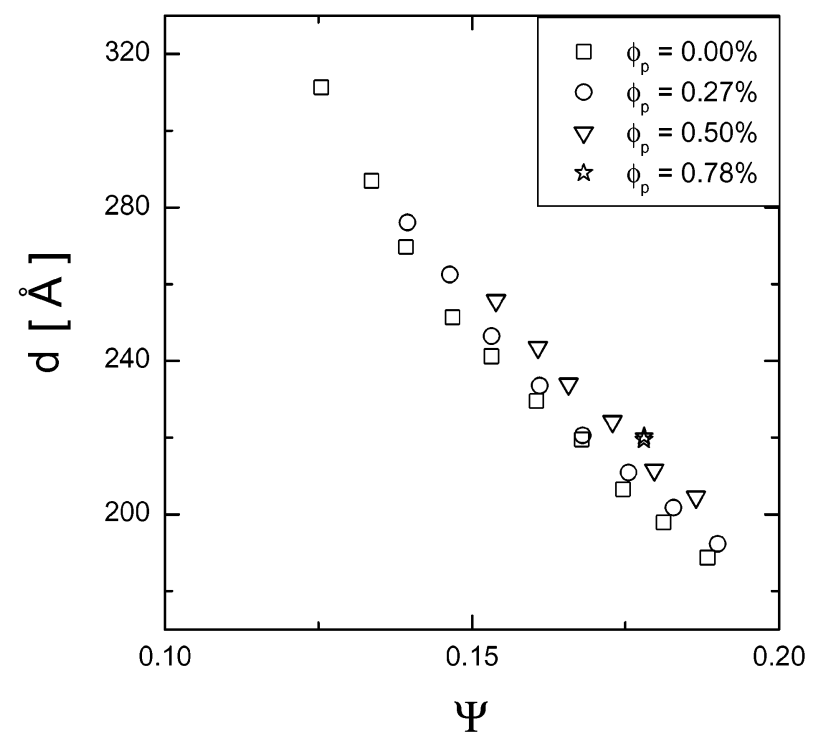

Figure 4. Domain size as a function of the membrane volume fraction for bicontinuous microemulsions with different homopolymer contents, $\phi_{\mathrm{p}}$. Values are obtained from TeubnerStrey model fittings.

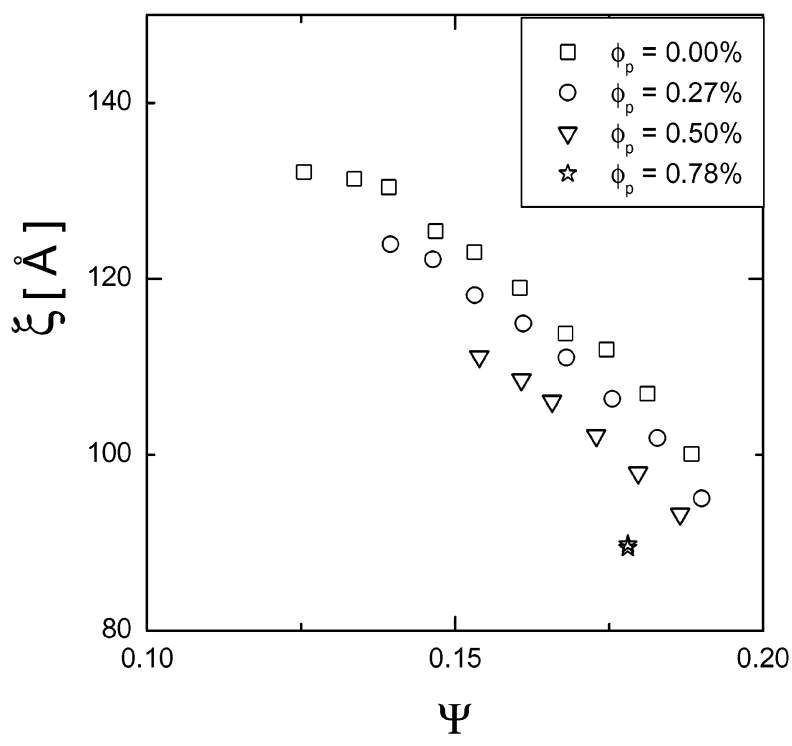

Figure 5. Correlation length as a function of the membrane volume fraction for bicontinuous microemulsions with different homopolymer contents, $\phi_{\mathrm{p}}$. Values are obtained from TeubnerStrey model fittings.

Neglecting fluctuations, one would expect the behavior $d$ $\sim \psi^{-1}$ in the limit of large structures. For large surfactant concentrations, membrane fluctuations can modify this dependence (see the Discussion).

Figure 5 represents the correlation length, $\xi$, as a function of homopolymer content and membrane volume fraction. The addition of homopolymer decreases the correlation length or, in other words, amplifies the membrane fluctuations. The decrease of $\xi$ with increasing membrane volume fraction, $\psi$, is related to the decrease of the domain size, $d$, with increasing $\psi$, as shown in Figure 4. While an increasing homopolymer volume fraction just slightly increases the domain size, $d$, the resulting decrease of $\xi$ is stronger pronounced, which is already an indication for a decreasing bending rigidity upon homopolymer addition.

The membrane bending rigidity, $\kappa_{\mathrm{R}}$, is calculated on the basis of the Gaussian random field theory for known 


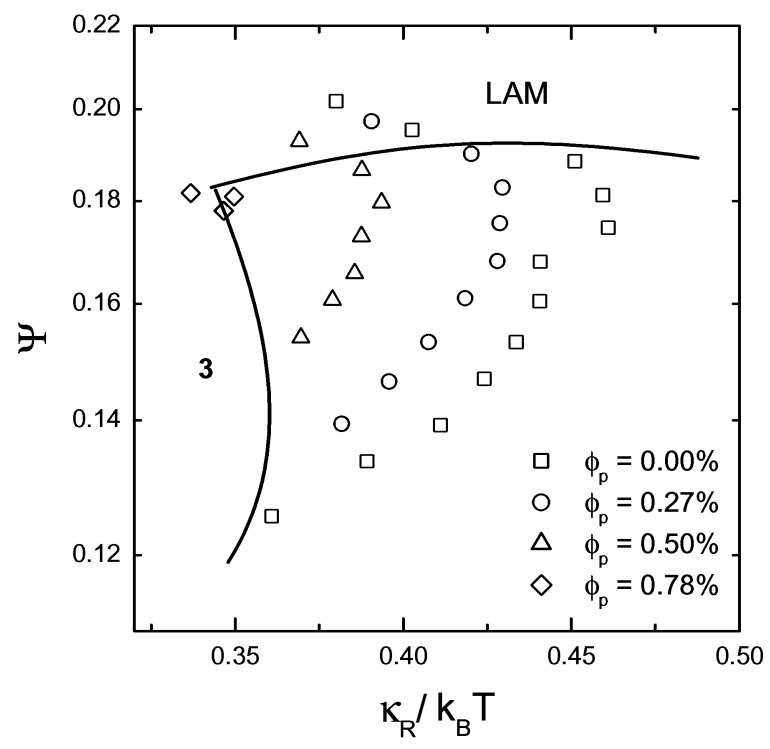

Figure 6. Membrane volume fraction as a function of the bending rigidity for different homopolymer volume fractions, $\phi_{\mathrm{p}}$. Solid lines separate regions of the three-phase coexistence (3) and the lamellar phase (LAM).

$d$ and $\xi$ (see eq 5). In Figure 6, the membrane volume fraction, $\psi$, at the fish-tail point is plotted as a function of this bending rigidity, $\kappa_{\mathrm{R}}$, for different homopolymer contents. In this figure, two phase boundaries are indicated by solid lines. At low $\kappa_{\mathrm{R}}$, there is the three-phase coexistence region; the boundary indicates the emulsification failure. At high membrane volume fractions, the lamellar phase appears. The lamellar phase is observed by visual inspection (turbidity) and a sudden change of the shape of the scattering curves. The turbidity could either arise from many birefringent nonoriented crystallites or from a narrow two-phase region between the bicontinuous and the actual lamellar phases. Since the current study focuses on the bicontinuous phase, no efforts were made to further analyze this lamellar region. The interdependence (eq 2) between the elastic constant, $\kappa_{R}$, membrane volume fraction, $\psi$, and homopolymer content, $\phi_{\mathrm{p}}$, provides a way to check the effect of thermal fluctuations on $\kappa_{\mathrm{R}}$. For the spatial renormalization, we get at constant homopolymer content

$$
\ln \left(\psi / \psi_{0}\right)=\frac{4 \pi \kappa_{\mathrm{R}}}{\alpha k_{\mathrm{B}} T}
$$

For the coefficient $\alpha$, an experimental value of $3.2 \pm 0.5$ was found, which agrees with the theoretical prediction $\alpha=3^{21,22}$ and previous measurements. ${ }^{3}$ The other dependence of the bending rigidity, $\kappa_{R}$, on the homopolymer content (see Figure 7) can be checked by focusing on the homopolymer term of eq 2 :

$$
\frac{\Delta \kappa_{\mathrm{R}}}{k_{\mathrm{B}} T}=-\beta \phi_{\mathrm{p}} \frac{\left(R_{\mathrm{hW}}^{3}+R_{\mathrm{hO}}^{3}\right)}{V_{\mathrm{p}}}
$$

The experimental coefficient $\beta=0.15 \pm 0.02$ is 6.3 times larger than the theoretically predicted value $\beta=0.0238 .{ }^{19}$ This discrepancy agrees with the approximately 7 times higher sensitivity of the phase diagram (see Figure 2) expressed by the coefficient $\bar{\beta}$. A possible origin of this discrepancy will be discussed below.

Simultaneous Addition of Homopolymers and Diblock Copolymers. A tunable viscosity and a nonreduced efficiency may be achieved by the simultaneous

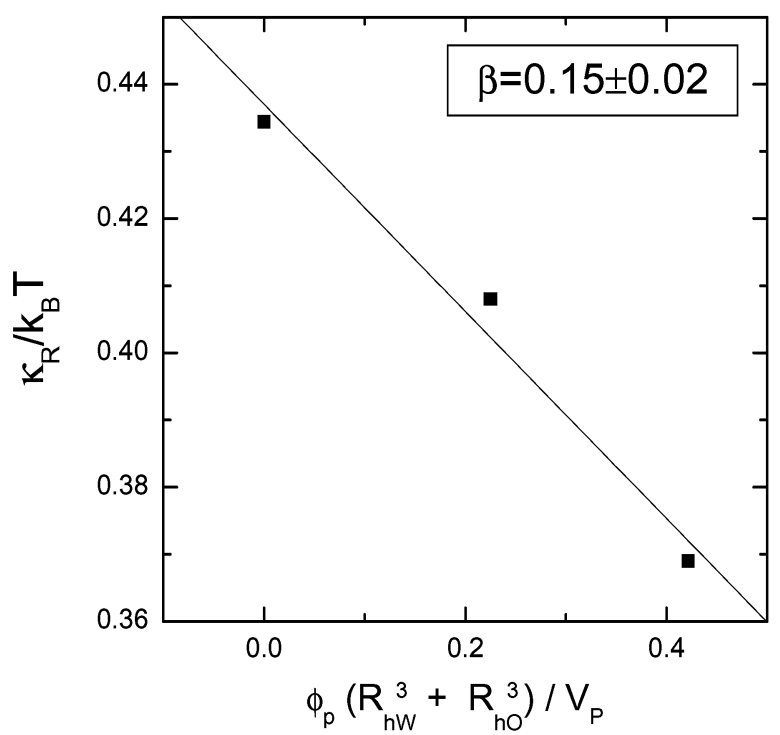

Figure 7. Renormalized bending rigidity as a function of the scaled homopolymer volume fraction. The slope of the regression line is indicated by $\beta$.

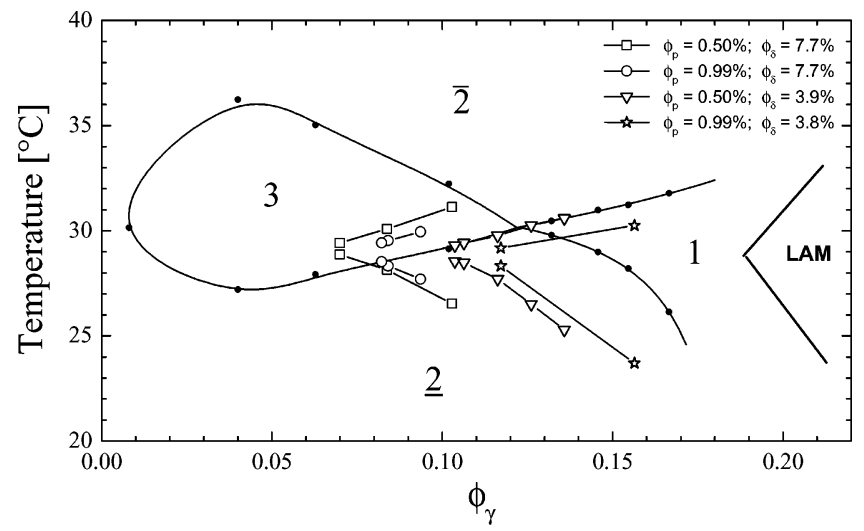

Figure 8. Phase diagram: Fish diagram (solid line, black filled dots) for the system $\mathrm{H}_{2} \mathrm{O}$-decane $-\mathrm{C}_{10} \mathrm{E}_{4}$. For homopolymer $\phi_{\mathrm{p}}$ and diblock copolymer $\phi_{\delta}$ added to the system $\mathrm{D}_{2} \mathrm{O}$-decane$\mathrm{C}_{10} \mathrm{E}_{4}$, lines with hollow symbols are used (see legend).

addition of homopolymers and diblock copolymers. This implies the superposition of the two polymer influences, as assumed in eq 2 . The polymers used in this study are the homopolymers $\mathrm{PEP}_{5}$ and $\mathrm{PEO}_{5}$ and the symmetric diblock copolymer $\mathrm{PEP}_{5}-\mathrm{PEO}_{5}$. Phase diagrams are shown in Figure 8. The more diblock copolymer is added, the more the fish-tail point moves to lower surfactant contents. The effect of homopolymers is opposite: For a given diblock copolymer content, the fish-tail point shifts to higher $\phi_{\gamma}$ with increasing homopolymer content. The two polymer additions have opposite effects on the surfactant efficiency. Homopolymers decrease and diblock copolymers increase the surfactant stabilization properties.

In the detailed analysis, we focus on the fish-tail points (solubilization failure) as a function of the two polymer contents. In Figure 2, the membrane volume fraction at the fish-tail point is plotted as a function of the homopolymer volume fraction for different diblock copolymer contents. For all curves, the membrane volume fraction increases with the homopolymer volume fraction, which means a decrease in the surfactant efficiency (compare Figure 2). The lines for certain amounts of added diblock copolymer are quite parallel. The slopes $\bar{\beta}_{1}=0.35$ and $\bar{\beta}_{2}$ $=0.33$ are comparable to $\bar{\beta}=0.54$ without diblock 


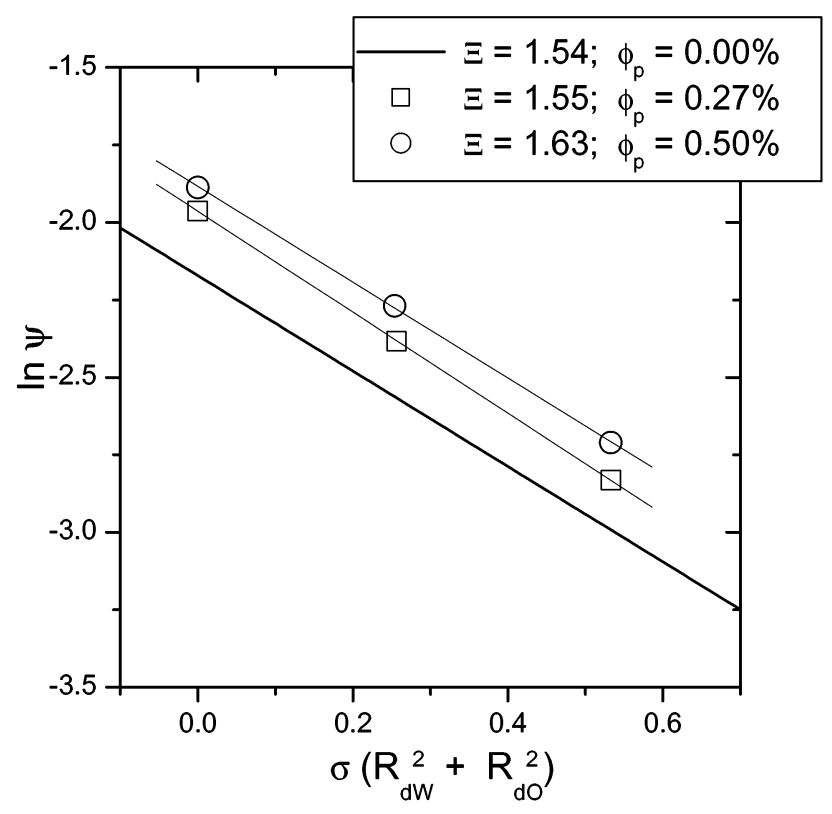

Figure 9. Logarithm of the membrane volume fraction at the fish-tail point as a function of the diblock copolymer grafting density. The different data sets arise from different amounts of homopolymers, $\phi_{\mathrm{p}}$, simultaneously added. The bottom solid line (no homopolymer) is taken from the literature. ${ }^{2,29}$

copolymer (see eq 6). This is already a hint for a possible superposition of the two opposite polymer effects.

While the previous analysis focused on the homopolymer content, the following analysis concerns the diblock copolymer addition. In the same manner as eq 6 , the dependence of the membrane volume fraction on the scaled diblock copolymer content is derived on the basis of eq 3 (see ref 2):

$$
\psi=\psi_{0} \exp \left[-\Xi \sigma\left(R_{\mathrm{dW}}^{2}+R_{\mathrm{dO}}^{2}\right)\right]
$$

In Figure 9, the dependence of the membrane volume fraction at the fish-tail point on the scaled polymer grafting density for samples with and without homopolymer is shown. For ideal polymer chains, the coefficient $\Xi=\pi / 5 \approx$ 0.63 is predicted. The experimental coefficient $t^{2} \Xi=1.54$ \pm 0.05 is larger however. The experimental coefficients of our study for a simultaneous constant homopolymer content are $\Xi_{1}=1.55 \pm 0.05$ and $\Xi_{2}=1.63 \pm 0.05$. This result fits very well with the results for homopolymerfree microemulsions. Therefore, it can be concluded that the addition of homopolymer does not influence the boosting effect of the diblock copolymer and that the two opposite effects really superpose.

Again, the effect of diblock copolymer addition to microemulsions can be discussed as the effect on the bending rigidity, $\kappa_{\mathrm{R}}$, and therefore be related to microemulsion structure size obtained by SANS measurements. All samples were measured under bulk contrast. The surfactant volume fraction was varied in the range from 7 to 20 vol \%. The homopolymers $\mathrm{PEP}_{5}$ and $\mathrm{PEO}_{5}$ were added to oil and water, respectively, in equal amounts with an increment of $\sim 0.25 \mathrm{vol} \%$. The diblock copolymer content of $\mathrm{PEP}_{5}-\mathrm{PEO}_{5}$ was chosen to be $\phi_{\delta} \approx 3.8$ vol $\%$ and $\phi_{\delta} \approx 7.7 \mathrm{vol} \%$. Figure 10 shows an example of the macroscopic cross section as a function of the scattering vector for selected polymer contents. The membrane volume fraction for the represented scattering curves was chosen to be $0.129 \pm 0.005$. For reasons of clarity, the cross section of different samples was consequently

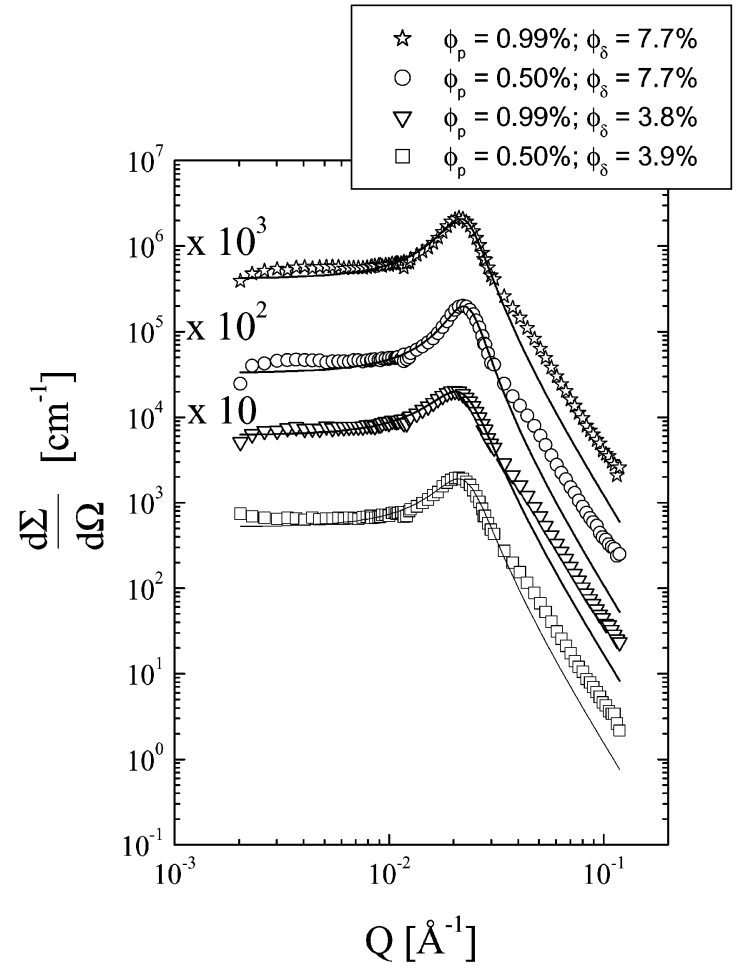

Figure 10. Scattering cross section as a function of the scattering vector for bicontinuous microemulsions at given volume fractions of homopolymers, $\phi_{\mathrm{p}}$, and diblock copolymer, $\phi_{\delta}$. The membrane volume fraction, $\psi$, is fixed at 0.129 . Errors are of the approximate symbol size or below. Solid lines are Teubner-Strey model fittings.

multiplied by a factor of 10. Qualitatively, it can be seen that the homopolymer makes the peak broader and less pronounced, which means a decrease of the correlation length. On the other hand, the diblock copolymer causes a sharper and more pronounced peak. Finally, our quantitative analysis of the scattering data is based on the Teubner-Strey formula (eq 4). Examples of fitting curves are also shown in Figure 10.

The resulting domain size, $d$, is plotted in Figure 11 as a function of the membrane volume fraction for samples with different homopolymer and diblock copolymer contents. The function $d=37 \AA \times \psi^{-1}$ describes the data reasonably well. This function is not sensitive to polymer additions but is characteristic for the surfactant. This universal behavior (no polymer influence) indicates that the surfactant dominates the structure formation.

Figure 12 represents the correlation length as a function of the membrane volume fraction for given homopolymer and diblock copolymer contents. The addition of homopolymer decreases the correlation length or increases the strength of membrane fluctuations. On the other hand, the addition of diblock copolymer causes an increase of the correlation length and suppresses fluctuations. From this plot, the superposition of the two opposite effects of polymer additions is clearly seen. The correlation length decreases with increasing membrane volume fraction. Again, the polymer effect mainly results from the bending rigidity, whereas the addition of surfactant causes a decrease of $\xi$, as $\xi$ scales with the structure size, $d$, which decreases with increasing membrane volume fraction (Figure 5).

In Figure 13, the membrane volume fraction is plotted as a function of the renormalized bending rigidity, $\kappa_{R}$, for several homopolymer contents and a fixed diblock copolymer content. As before, the regions of the lamellar 


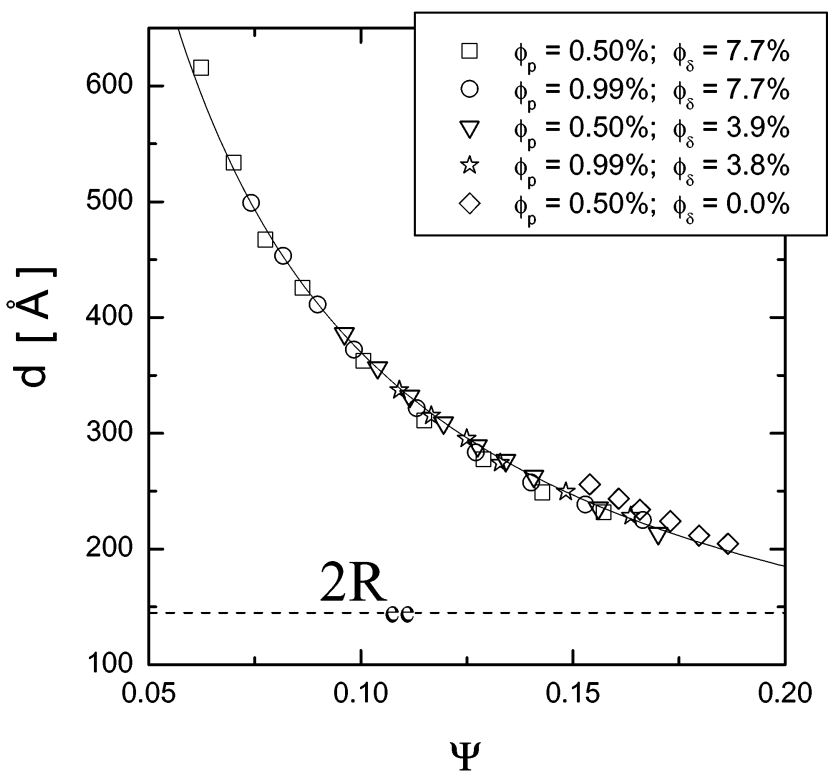

Figure 11. Domain size as a function of the membrane volume fraction for bicontinuous microemulsions with different homopolymer, $\phi_{\mathrm{p}}$, and diblock copolymer, $\phi_{\delta}$, contents. Values are obtained from Teubner-Strey model fittings. The obtained simple dependence (solid line, $d=37 \AA / \psi$ ) shows that only the amount of surfactant is responsible for the structure. The double homopolymer end-to-end distance, $2 R_{\text {ee }}$, is indicated by the dashed line (see the Discussion).

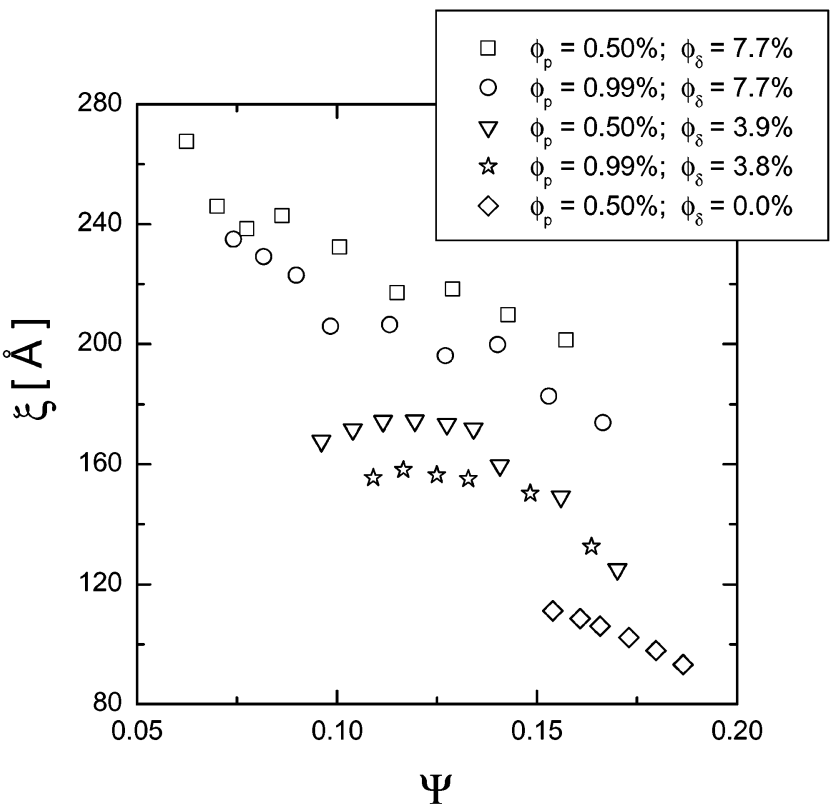

Figure 12. Correlation length as a function of the membrane volume fraction for bicontinuous microemulsions with different homopolymer, $\phi_{\mathrm{p}}$, and diblock copolymer, $\phi_{\delta}$, contents. Values are obtained from Teubner-Strey model fittings.

and the three-phase coexistence are outlined. The solid line for a microemulsion without homopolymer is calculated on the basis of the experimental values obtained in ref 29. The difference of the slopes between this study and previous measurements at low $\psi$ can be caused by increased fluctuations due to the homopolymer addition. The sensitivity of $\kappa_{\mathrm{R}}$ on the homopolymer content can be roughly estimated by horizontal cuts in these plots (compare Figures 6 and 7 and eq 8). From the plots of Figure 13, a rough estimate of $\beta=0.16 \pm 0.02$ is obtained. This agrees well with the previous result without diblock copolymer $(\beta=0.15 \pm 0.02)$.

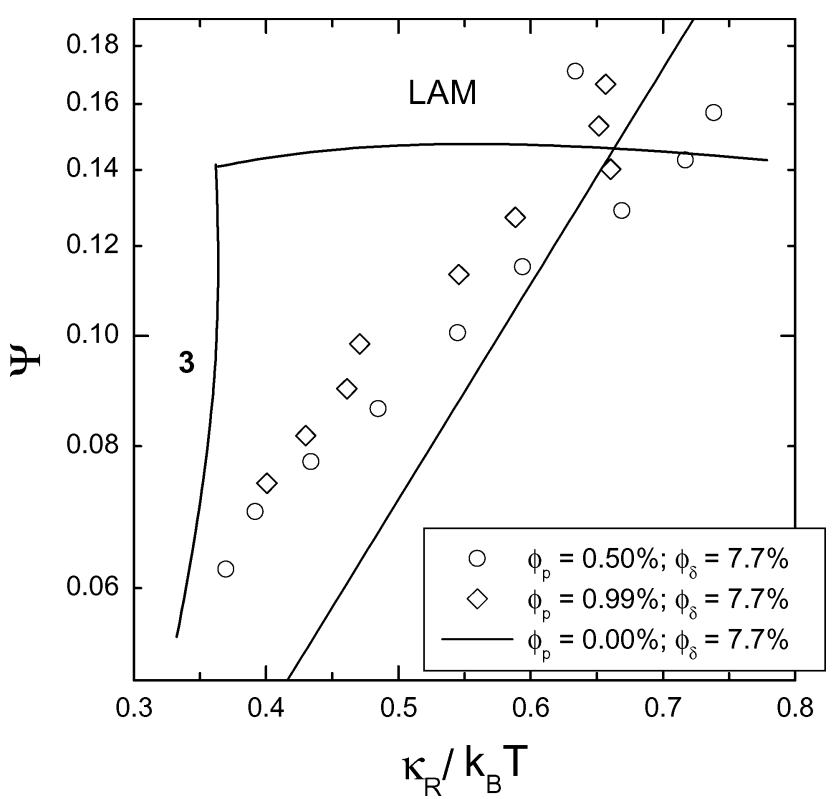

Figure 13. Membrane volume fraction as a function of the bending rigidity for different amounts of homopolymer, $\phi_{\mathrm{p}}$, and high amounts of diblock copolymer $\left(\phi_{\delta}=7.7 \%\right)$. The straight line is calculated for a microemulsion without homopolymer. ${ }^{29}$

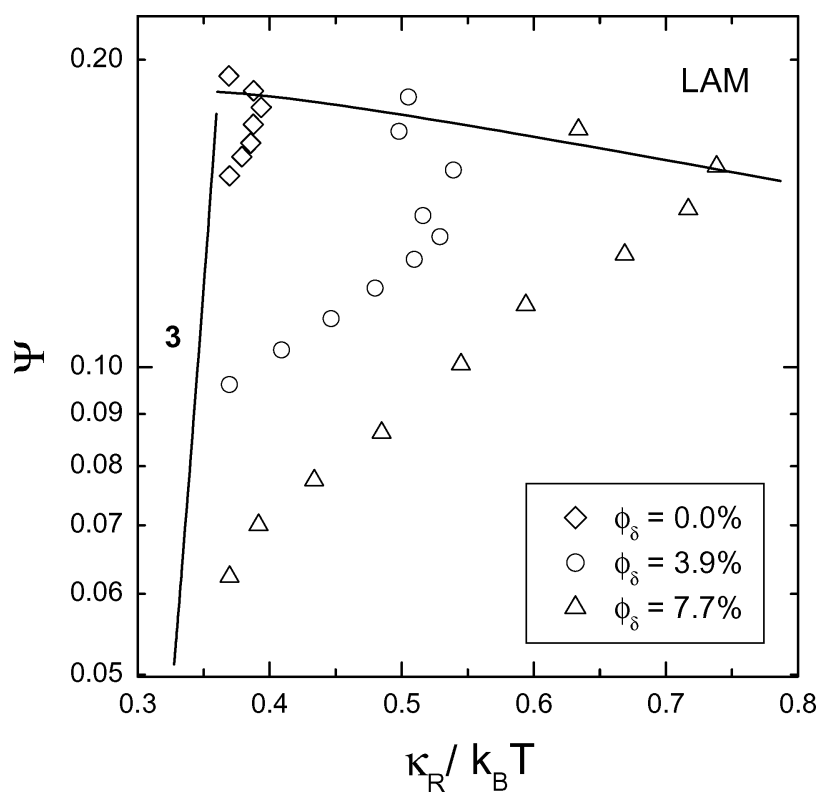

Figure 14. Membrane volume fraction as a function of the bending rigidity for different amounts of diblock copolymer, $\phi_{\delta}$, and an intermediate amount of homopolymer $\left(\phi_{\mathrm{p}}=0.5 \%\right)$.

The dependence of $\psi$ on $\kappa_{\mathrm{R}}$ is shown in Figure 14 for different amounts of diblock copolymer with a fixed homopolymer content. A horizontal cut yields the dependence of the bending rigidity on the diblock copolymer content. The corresponding expression can be derived from eq 2:

$$
\frac{\Delta \kappa_{\mathrm{R}}}{k_{\mathrm{B}} T}=-\hat{\Xi} \sigma\left(R_{\mathrm{dW}}^{2}+R_{\mathrm{dO}}^{2}\right)
$$

The coefficient $\hat{\Xi}$ is obtained for two different constant amounts of homopolymer, $\hat{\Xi}_{1} \approx 0.41$ and $\hat{\Xi}_{2} \approx 0.43$. Both values agree quite well with the literature value $\hat{\Xi}=$ $0.334 .^{26}$

The gap of $\kappa_{\mathrm{R}}$ at low membrane volume fraction, $\psi$, remains an open question. This does not correspond to 
the phase diagrams. Nonetheless, once a certain amount of homopolymer $(\sim 0.5 \mathrm{vol} \%)$ is added, the dependence of $\kappa_{\mathrm{R}}$ seems to behave normally. This means that the extracted $\beta$ and $\Xi$ values of two-kind polymer additions correspond to polymer additions of one kind. The supposed superposition of the two opposite effects of polymer additions is at least partially fulfilled.

\section{Discussion}

While in previous studies ${ }^{3,29}$ eq 5 was used to obtain the bending rigidity, another option is to calculate the bending rigidity from $\xi$ values obtained by SANS measurements. The Gaussian random fields model ${ }^{28,29}$ yields the formula $\xi S / V=(128 / 15 \pi) \kappa_{\mathrm{R}}$ within a first approximation. The surfaceto-volume ratio is calculated from the membrane volume fraction and the membrane thickness $\delta=12 \AA . .^{26,29}$ Comparing both data sets with different amounts of homopolymers but without diblock copolymer, one finds that the spanned range of $\kappa_{\mathrm{R}}$ has a width of $\Delta \kappa_{\mathrm{R}} \approx 0.11 k_{\mathrm{B}} T$ in either case; just the absolute values are shifted (like $0.42 \rightarrow 0.57$ ). This discrepancy is due to higher order terms such as the function $\Theta$ in eq 5 . At the present time, we prefer the already used formula for reasons of comparison.

For the domain size, a simple dependence was found for a wide range of membrane volume fractions (see Figure 11). The large domain size, $d$, does not seem to be dependent on the polymer type and amount, while at small domain sizes the homopolymer caused an observable effect (see Figure 4). An expression for the domain size with fluctuations was given by Roux et al. ${ }^{32}$ based on a theory by Helfrich: ${ }^{17}$

$$
d=\frac{2 \delta}{\psi}\left(1+\frac{1}{4 \pi} \frac{k_{\mathrm{B}} T}{\kappa_{\mathrm{R}}} \ln \left(c \sqrt{\frac{\kappa_{\mathrm{R}}}{k_{\mathrm{B}} T}} \frac{d}{2 a}\right)\right)
$$

$c=1.84$ is a constant for the practical cutoff. $a \approx 7 \AA$ is connected with the area $\left(53.9 \AA^{233}\right)$ per surfactant molecule. The first factor corresponds to a lamellar structure without fluctuations, and the bracket describes fluctuation corrections for lamellar and/or bicontinuous structures. If the whole bracket is assumed to be constant, one gets $d=3.2 \delta / \psi=38 \AA / \psi$ (using $d=251 \AA$ and $\kappa_{\mathrm{R}}=$ $0.42 k_{\mathrm{B}} T$ ). This compares well with the overall experimentally found behavior (coefficient $37 \AA$ ), that is, with mainly large $d$.

A more detailed discussion on eq 11 can be made with the full dependence of $d$ on $\kappa_{\mathrm{R}}$. Experimentally, the scaled $d$ increases by $8.6 \%$ (see Figure 4 ) with the highest amount of homopolymer. The bending rigidity decreases by $30 \%$ (see Figure 6). Theoretically, this corresponds to an increase of $d$ by $11.5 \%$. This means that the domain size is influenced by membrane fluctuations, which is clearly observed for high surfactant amounts (and, here, high polymer contents). The fluctuations become much less important for large domain sizes. The reason is that the diblock copolymer allows for large $d$ by increasing the bending rigidity (see the spanned $\kappa_{\mathrm{R}}$ range in Figure 13).

(31) End-to-end distances were calculated from the following references. PEP in benzene: Mays, J. M.; Fetters, L. J. Macromolecules 1989, 22, 921. PEP in cyclohexane (preferred): Fetters, L. J. Private communication calculated from viscosities. PEO in water: Kawaguchi, S.; Imai, G.; Suzuki, J.; Miyahara, A.; Kitano, T.; Ito, K. Polymer 1997, 38,2885 .

(32) Roux, D.; Nallet, F.; Freyssingeas, E.; Porte, G.; Bassereau, P.; Skouri, M.; Marignan, J. Europhys. Lett. 1992, 17, 575.

(33) Sottmann, T.; Strey, R.; Chen, S.-H. J. Chem. Phys. 1997, 106, 6483. Sottmann, T. Ph.D. Dissertation, Georg-August-Universität zu Göttingen, Göttingen, Germany, 1997.
Now we focus on the polymer dependence of the bending rigidity, $\kappa_{R}$, and the saddle splay modulus, $\bar{\kappa}_{\mathrm{R}}$ : (a) The experimental result is that the value $\bar{\beta}$ expresses an approximately 7 times higher sensitivity on the homopolymer content than the theory of Eisenriegler predicts (Figure 2). Similarly, $\beta$ is 6.3 times larger than expected (Figure 7). Therefore, both coefficients express the same higher sensitivity. (b) The dependence of $\kappa_{\mathrm{R}}$ shows a discontinuity between no homopolymer and minimum homopolymer amount at low surfactant concentrations (Figure 13). Furthermore, the values of $\bar{\beta}$ are slightly dependent on the presence of diblock copolymer (Figure 2 ). The presence of one and the other polymer slightly changes the behavior of the microemulsion. However, these changes are so small that we still claim that the effects of the two polymer types superpose. (c) The sensitivity of the phase diagram on the diblock copolymer content ( $\Xi$, see Figure 9 ) does not depend on the presence of homopolymer. The dependence of $\kappa_{\mathrm{R}}$ on the diblock copolymer content (Figure 14) seems to be continuous. Therefore, the $\hat{\Xi}$ coefficients agree well for different homopolymer concentrations.

Now, the sophisticated theory of Eisenriegler ${ }^{19}$ describing the influence of homopolymers on membranes should be compared with another similar model and with a model describing a different effect. The Eisenriegler theory describes the polymer depletion near surfaces, which results in entropic contributions that describe a floppier membrane surface. The theory of Kabalnov ${ }^{34}$ models membranes of finite thickness in the presence of a component dissolved in the bulk (here, ions). It is assumed that a depletion zone exists which is connected to an osmotic pressure. Using the typical depletion zone $\left(R_{\mathrm{g}}\right)$ of a polymer and the osmotic pressure of polymers in solution (equivalent to an ideal gas), one ends up with a quantitatively very similar formula for the saddle splay modulus, $\Delta \bar{\kappa}_{\mathrm{R}}$. One should be cautious with membrane finite size effects, since Kabalnov's theory compares the state of a membrane with thickness $h$ in the presence of the solute with the state of an infinitely thin membrane without solute. The comparison of Eisenriegler's theory refers to an infinitely thin membrane in either case. Finally, Eisenriegler's theory should be preferred over Kabalnov's, since both theories basically describe the same effect but Eisenriegler's theory finds also a valuable expression for the change of the bending rigidity, $\Delta \kappa_{R}$, which is not negligible. The theory of Brooks ${ }^{35}$ considers adsorbed polymer in the vicinity of membranes. The sign of the changes of the two moduli, $\Delta \kappa_{R}$ and $\Delta \bar{\kappa}_{R}$, is the same as that in the theories without adsorption. However, in the case of weak adsorption, the changes $\Delta \kappa_{R}$ and $\Delta \bar{\kappa}_{R}$ scale as $\phi_{\mathrm{p}}^{-1 / 2}$ and even as $\phi_{\mathrm{p}}^{-1}$ in the case of very strong adsorption. In our case, this behavior is not found (see Figure 7). Since we studied symmetric systems, we did not compare predictions of the spontaneous curvature.

A first trial explanation of the high sensitivity on the homopolymer content might be given by a zone of repulsion (depletion zone) for the homopolymer. Typically, the width of this zone agrees with the radius of gyration, which leaves a smaller available volume for homopolymers. The remaining space, $(d / 2)-2 R_{\mathrm{g}} \approx 125-57 \AA$, measures approximatively half of the distance $d / 2$. This factor would yield a 2 times higher effective polymer concentration, which does not fully explain the 7 times higher sensitivity.

(34) Kabalnov, A.; Olsson, U.; Wennerström, H. J. Phys. Chem. 1995, 99,6220 .

(35) Brooks, J. T.; Marques, C. M.; Cates, M. E. J. Phys. II 1991, 1, 


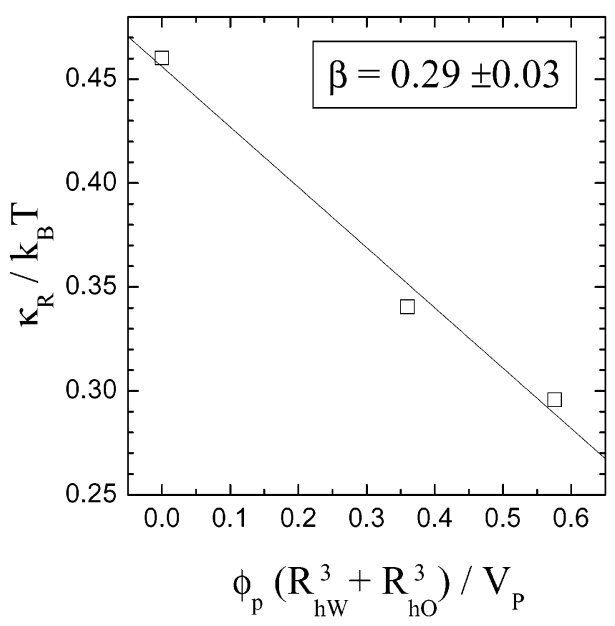

Figure 15. Renormalized bending rigidity as a function of the scaled homopolymer volume fraction. The higher molar mass of the polymer leads to an approximately 2 times higher slope, $\beta$.

Another explanation of the strong sensitivity on the homopolymer content might be given by the studies of Auth. ${ }^{36}$ One should compare the end-to-end distance of one dissolved block or homopolymer $\left(R_{\text {ee }} \approx 70 \AA\right)$ with the minimum domain size, $d / 2 \approx 100 \AA$ (see Figure 11 ). The polymer is not really confined by the domains, but the polymer is quite close to confinement. Following the simulations of ref 36 , the influence of a diblock copolymer on the bending rigidity, $\Delta \kappa_{\mathrm{R}}$, for a confined geometry behaves as follows: For large $d$, one obtains the classical value (eq 2 ). With values of $d / 2 \approx R_{\mathrm{g}}$ (close to the radius of gyration! $R_{\mathrm{g}}=R_{\mathrm{ee}} /(6)^{1 / 2} \approx 29 \AA$ ), Auth finds a maximum value of $\Delta \kappa_{\mathrm{R}}$ several times bigger than the classical value. For strongly confined geometries, $\Delta \kappa_{\mathrm{R}}$ quite quickly becomes negative. This strong decrease of $\Delta \kappa_{\mathrm{R}}$ is explained by the response of the polymer (monomer distribution) on the curved surface. This response gives a negative contribution to the coefficient for the curvature squared term of the free energy, which means a negative bending rigidity contribution, $\Delta \kappa_{\mathrm{R}}$. For highly confined polymers, there should be no difference between anchored polymers (diblock copolymers) and homopolymers. Therefore, $\Delta \kappa_{\mathrm{R}}$ of homopolymers would behave as follows: For large $d$, one would obtain the classical negative value of Eisenriegler, and with decreasing $d, \Delta \kappa_{\mathrm{R}}$ would become more and more negative. These highly negative values would, in principle, explain the high sensitivity of phase diagrams and the bending rigidity on the homopolymer content $\left(\kappa_{\mathrm{R}}\right.$, $\bar{\kappa}_{\mathrm{R}}$ ). In a detailed discussion, one might find explanations for the apparent "discontinuities" of $\kappa_{\mathrm{R}}$. Just to repeat, the only uncertainty is the range of confinement $(d / 2)$, when the scenario described above becomes dominating.

Another example shows that homopolymers with higher molecular weight $\mathrm{PEP}_{10}$ and $\mathrm{PEO}_{10}$ are responsible for an even higher sensitivity of $\kappa_{R}$ and $\bar{\kappa}_{R}$. This result would perfectly fit the theoretical picture drawn. The membrane volume fraction at the fish-tail point as a function of the scaled homopolymer content (not shown here) yields the coefficient $\beta=0.72$, which is $33 \%$ larger than the corresponding value with lower molecular weight. In Figure 15, the bending rigidity, $\kappa_{\mathrm{R}}$, is plotted as a function of the scaled homopolymer content. The extracted value of $\beta=0.29$ is approximately 2 times higher than the reference value $\beta=0.15$. These results demonstrate a higher sensitivity of $\kappa_{\mathrm{R}}$ and $\bar{\kappa}_{\mathrm{R}}$ with stronger confinement.

(36) Auth, T. Ph.D. Dissertation, Universität Köln, Köln, Germany, 2004 (and private communications).
The depletion zone explanation and the confined polymer explanation can be distinguished. On one hand, the polymer is repelled by the membrane, whereas it is not strongly repelled on the other hand. This question could be solved by detailed contrast variation SANS experiments.

\section{Summary and Conclusions}

Microemulsions consisting of water, oil, nonionic surfactant, and various polymers were investigated by two complementary methods: the macroscopic phase behavior was studied, and the corresponding microscopic structure was obtained by SANS measurements. The main aim of our work was to get a deeper insight on the effects caused by small added amounts of polymers to the microemulsions. After the polymer boosting effect has been established-this is a strong enhancement of the emulsification behavior by the addition of amphiphilic diblock copolymer-the complementary effect of homopolymers was under consideration now. Simultaneous additions of homopolymers and diblock copolymer were investigated to check if the two opposite effects are superposable and if one can get a constant efficiency with changed viscosity.

First, homopolymers were added to the bicontinuous microemulsion only. Equal amounts of water- and oilsoluble polymers with the same molecular weight were used. The phase behavior in this case demonstrated a diminished efficiency of the surfactant. The sensitivity of the phase diagram on the homopolymer addition is connected with the saddle splay modulus and therefore can be compared with theory. By SANS, we measured the microscopic structure of the microemulsion. Describing the scattering data with the Teubner-Strey formula, we obtained the structural parameters of the microemulsion: the characteristic domain size, $d$, and the correlation length, $\xi$. The Gaussian random field model allows extraction of the elastic modulus, $\kappa_{\mathrm{R}}$, from the structural parameters. A decrease of the bending rigidity, $\kappa_{\mathrm{R}}$, was observed with increasing homopolymer content. The sensitivity of both the emulsification ability and of $\kappa_{\mathrm{R}}$ on the homopolymer content is about 7 times stronger than that predicted by theory. In the future, it has to be discussed whether the confinement of the polymer between the membranes is responsible for this high sensitivity.

Then, the simultaneous addition of homopolymers and diblock copolymer was explored. The phase diagram indicates that the diblock copolymer increases the efficiency while the homopolymer decreases it. The connection of the emulsification boundary with the saddle splay modulus, $\bar{\kappa}_{\mathrm{R}}$, leads to the deduction that $\bar{\kappa}_{\mathrm{R}}$ increases by the homopolymer addition and decreases by the addition of diblock copolymer. Again, the SANS measurements allow for the determination of the structural parameters $d$ and $\xi$. The bending modulus, $\kappa_{\mathrm{R}}$, is calculated. Addition of the homopolymer decreases $\kappa_{R}$, while the addition of diblock increases it. For this simultaneous addition of polymers, the sensitivity of $\kappa_{\mathrm{R}}$ and $\bar{\kappa}_{\mathrm{R}}$ on the homopolymer addition is the same as in the case without diblock copolymer. Also, the effect of diblock copolymer addition is in good agreement with results obtained in a previous work. ${ }^{2,29}$ The conclusion is that these two opposite effects act independently in the studied range of polymer concentrations. Thus, this behavior allows for the change of viscosity at constant surfactant efficiency.

Acknowledgment. We would like to thank M. Monkenbusch for critical reading of the manuscript. 
Fruitful discussions with G. Gompper increased the quality of this work.

\section{Appendix-Variables}

$a$ : diameter that one surfactant molecule occupies in plane $c$ : cutoff parameter in theory of Roux ${ }^{32}$

$c_{0}$ : spontaneous curvature

$c_{1}, c_{2}$ : principal curvatures

$d$ : domain size

$F$ : free energy

g: genus of surface

$H$ : mean curvature

$I$ : scattering intensity $(\sim(\mathrm{d} \Sigma / \mathrm{d} \Omega))$

$k_{0}$ : domain size wave vector

$k_{\mathrm{B}} T$ : thermal energy

$K$ : Gaussian curvature

$n$ : number of separated bodies

$Q$ : scattering vector

$Q_{\max }$ : scattering vector of maximum intensity

$Q_{P}$ : scattering vector separating Teubner-Strey from

Porod region

$R_{1}, R_{2}$ : principal radii

$R_{\mathrm{dW}}$ : end-to-end distance of one diblock copolymer block in water domains

$R_{\mathrm{do}}$ : end-to-end distance of one diblock copolymer block in oil domains

$R_{\text {ee }}$ : end-to-end distance in general

$R_{\mathrm{g}}$ : radius of gyration in general

$R_{\mathrm{hW}}$ : end-to-end distance of homopolymer in water domains

$R_{\mathrm{ho}}$ : end-to-end distance of homopolymer in oil domains

$S:$ membrane surface

$\mathrm{d} S$ : surface element

$V$ : microemulsion volume

$V_{\text {water: }}$ : sample volume of water fraction

$V_{\text {oil }}$ : sample volume of oil fraction

$V_{\text {surf: }}$ sample volume of surfactant fraction

$V_{\mathrm{hom}}^{\mathrm{O}}$ : sample volume of homopolymer in oil domains

$V_{\text {hom }}^{\mathrm{W}}$ : sample volume of homopolymer in water domains

$V_{\text {dibl }}$ : sample volume of dilock copolymer

$V_{\mathrm{p}}$ : homopolymer molar volume

$\alpha$ : coefficient for spatial renormalization of bending rigidity $\bar{\alpha}$ : coefficient for spatial renormalization of saddle splay modulus

$\beta$ : coefficient for homopolymer effect on bending rigidity

$\bar{\beta}$ : coefficient for homopolymer effect on saddle splay modulus

$\delta$ : thickness of surfactant membrane

$\Theta$ : correction between bending rigidity and structural parameters $\xi$ and $d$

$\kappa$ : microscopic bending rigidity

$\kappa_{\mathrm{R}}$ : renormalized bending rigidity

$\kappa_{0}$ : genuine bending rigidity (no polymer, $\psi$ extrapolated to 1 )

$\Delta \kappa_{\mathrm{R}}$ : change of bending rigidity due to polymer addition

$\bar{\kappa}$ : microscopic saddle splay modulus

$\bar{\kappa}_{\mathrm{R}}$ : renormalized saddle splay modulus

$\bar{\kappa}_{0}$ : genuine saddle splay modulus (no polymer, $\psi$ extrapolated to 1 )

$\bar{\kappa}_{\text {FTP: }}$ saddle splay modulus at the fish-tail point

$\Delta \bar{\kappa}_{R}$ : change of saddle splay modulus due to polymer addition

$\lambda$ : neutron wavelength

$\Delta \lambda$ : neutron wavelength distribution ( $\mathrm{fwhm}$ )

$\left\langle v^{2}\right\rangle$ : fluctuation of scattering length density in domains

$\xi$ : correlation length

$\hat{\Xi}$ : coefficient for diblock copolymer effect on bending rigidity

$\Xi$ : coefficient for diblock copolymer effect on saddle splay modulus

$\sigma$ : grafting density of diblock copolymer on surfactant membrane

$\mathrm{d} \Sigma / \mathrm{d} \Omega$ : macroscopic scattering cross section

$\phi_{\mathrm{p}}$ : homopolymer content

$\phi_{\mathrm{O}}:$ volume ratio oil/(oil + water)

$\phi_{\gamma}$ : surfactant volume fraction

$\phi_{\delta}$ : volume fraction of diblock copolymer in total amphiphile

$\psi$ : membrane volume fraction

$\psi_{0}$ : membrane volume fraction at the fish-tail point without any polymer

LA049317T 